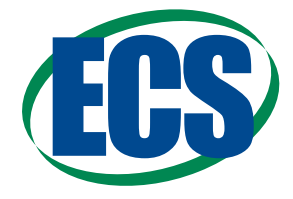

Jes Focus Issue on Semiconductor Electrochemistry and Photoelectrochemistry in Honor of Krishnan Rajeshwar

\title{
Integrated Membrane-Electrode-Assembly Photoelectrochemical Cell under Various Feed Conditions for Solar Water Splitting
}

\author{
Tobias A. Kistler, $\oplus^{1,2,3,4}$ David Larson, ${ }^{2,3}$ Karl Walczak, ${ }^{2,3, a}$ Peter Agbo, ${ }^{2,3}$ Ian D. Sharp,, ,3,b \\ Adam Z. Weber, ${ }^{1,3, *, z}$ and Nemanja Danilovic ${ }^{1}$ \\ ${ }^{I}$ Energy Storage and Distributed Resources Division, Lawrence Berkeley National Laboratory, Berkeley, \\ California, USA \\ ${ }^{2}$ Chemical Sciences Division, Lawrence Berkeley National Laboratory, Berkeley, California, USA \\ ${ }^{3}$ Joint Center for Artificial Photosynthesis, Lawrence Berkeley National Laboratory, Berkeley, California, USA \\ ${ }^{4}$ Chair of Technical Electrochemistry, Department of Chemistry, Technische Universität München, Munich, \\ Germany, USA
}

\begin{abstract}
Photoelectrochemical (PEC) water splitting has the potential to significantly reduce the costs associated with electrochemical hydrogen production through the direct utilization of solar energy. Many PEC cells utilize liquid electrolytes that are detrimental to the durability of the photovoltaic (PV) or photoactive materials at the heart of the device. The membrane-electrode-assembly (MEA) style, PEC cell presented herein is a deviation from that paradigm as a solid electrolyte is used, which allows the use of a water vapor feed. The result of this is a correspondent reduction in the amount of liquid and electrolyte contact with the PV, thereby opening the possibility of longer PEC device lifetimes. In this study, we demonstrate the operation of a liquid and vapor-fed PEC device utilizing a commercial III-V photovoltaic that achieves a solar-to-hydrogen (STH) efficiency of $7.5 \%$ (12\% as a PV-electrolyzer). While device longevity using liquid water was limited to less than 24 hours, replacement of reactant with water vapor permitted 100 hours of continuous operation under steady-state conditions and diurnal cycling. Key findings include the observations that the exposure of bulk water or water vapor to the PV must be minimized, and that operating in mass-transport limited regime gave preferable performance.

(C) The Author(s) 2018. Published by ECS. This is an open access article distributed under the terms of the Creative Commons Attribution 4.0 License (CC BY, http://creativecommons.org/licenses/by/4.0/), which permits unrestricted reuse of the work in any medium, provided the original work is properly cited. [DOI: 10.1149/2.0041905jes]

(cc) BY
\end{abstract}

Manuscript submitted October 15, 2018; revised manuscript received November 27, 2018. Published December 10, 2018. This paper

is part of the JES Focus Issue on Semiconductor Electrochemistry and Photoelectrochemistry in Honor of Krishnan Rajeshwar.

Widespread adoption of inexpensive renewable-electricity sources brings with it many exciting challenges and opportunities, the most significant being an overabundance of low-cost electricity as a result of low-cost solar panels and wind turbines. ${ }^{1,2}$ Thus, there is an opportunity to use virtually free electrons and photovoltaics to produce fuels and chemicals using electrochemical and photoelectrochemical devices, respectively with water and/or carbon dioxide as reactants. While electrochemical hydrogen production using water electrolysis is a commercial technology, barriers remain in terms of cost and efficiency improvements for its widespread adoption. ${ }^{3,4}$ Alternative water-splitting technologies that have the long-term potential to produce inexpensive hydrogen include photoelectrochemical (PEC) approaches, which are still in their infancy. ${ }^{5,6}$ A PEC cell comprises at least one and sometimes two photoelectrodes that provide a platform for direct solar-to-chemical conversion. The anodic reaction is oxygen evolution, while the cathodic reaction is hydrogen evolution. While there are many cell designs used for demonstrating material and device properties, a true PEC must have at least one PV-electrolyte junction, otherwise it is a solar driven electrochemical cell (PV-E). ${ }^{7-9}$ Most PEC studies and proposed devices consist of a liquid junction, in which a liquid electrolyte is in contact with one or both photoactive electrodes. ${ }^{10-14}$ The main disadvantage of the use of liquid electrolytes is that the $\mathrm{pH}$ is usually at $\mathrm{pH} 0$ or $\mathrm{pH} 14$, and degrades the semiconductor material at an unacceptably high rate through corrosion and photocorrosion mechanisms. ${ }^{15-17}$ Furthermore, the use of liquid feeds necessitates more complicated water management and piping at scale. One solution to the limited durability issue is to remove the liquid electrolyte and use liquid water or vapor in combination with a solid polymer electrolyte, such as Nafion. ${ }^{18-21}$ Herein, we describe a protonexchange-membrane (PEM)-based water-splitting platform (Figure 1)

\footnotetext{
*Electrochemical Society Fellow.

${ }^{\text {a }}$ Present address: Sandia National Laboratory, Albuquerque, New Mexico, USA.

${ }^{b}$ Present address: Walter Schottky Institute and Physics Department, Technische Universität München, Munich, Germany.

${ }^{\mathrm{z}}$ E-mail: azweber@lbl.gov
}

that can be used for PV-electrolysis (PV-E) and PEC operation with either liquid or vapor reactants, and that can easily accommodate photoanodes and photocathodes while producing stable solar to hydrogen efficiencies exceeding $12 \%$ for more than 100 hours.

\section{Experimental}

Cell architecture and components.-The philosophy behind the design of the cell includes many facets incorporated from traditional electrochemical membrane electrode assembly (MEA) based electrolyzers and fuel cells, which consist of endplates, flowfields, current collectors, and electrodes separated by an ion-conducting membrane. The main modifications that are introduced arise from the requirement that the photoactive components of the cell must have access to light. Due to this constraint, the endplates used herein are machined from polymethylmethacrylate (PMMA, McMaster-Carr Supply Company, Santa Fe Springs, CA), a tough, translucent, and chemically resistant plastic that ensures the mechanical sealing of the cell (Figure 1). The flow ports are attached with a two-component epoxy (EPO-TEK 302$3 \mathrm{M}$ ) in the holes of the plates (green tubes, perpendicular to endplate in Figure 1). A header is machined in the PMMA to improve the distribution of the reactant coming in and out of the endplate through the flow port, while decreasing the pump pressure. The endplates are used to compress the cell components, but since they are electrically isolating, a conductive tantalum foil with channels serves as a flowfield. The flowfields are used to channel reactants to the active sites and remove products, while also providing current collection. Furthermore, the flow channels serve as catalyst supports. The flowfields were fabricated using electrical discharge machining (EDM) or laser cutting $100 \mu \mathrm{m}$ thick tantalum foil, creating $0.9 \mathrm{~mm}$ wide channels, $100 \mu \mathrm{m}$ wide lands and channel lengths of $17.3 \mathrm{~mm}$. This pattern maximized the amount of light going through the cell, while still supporting the membrane and allowing for mass transport, which is very important for the photocathode (Figure 1a) configuration, in which the total illuminated PV area is limited by the tantalum foils. For the photoanode configuration (Figure 1b) the entire available area can be coated with 

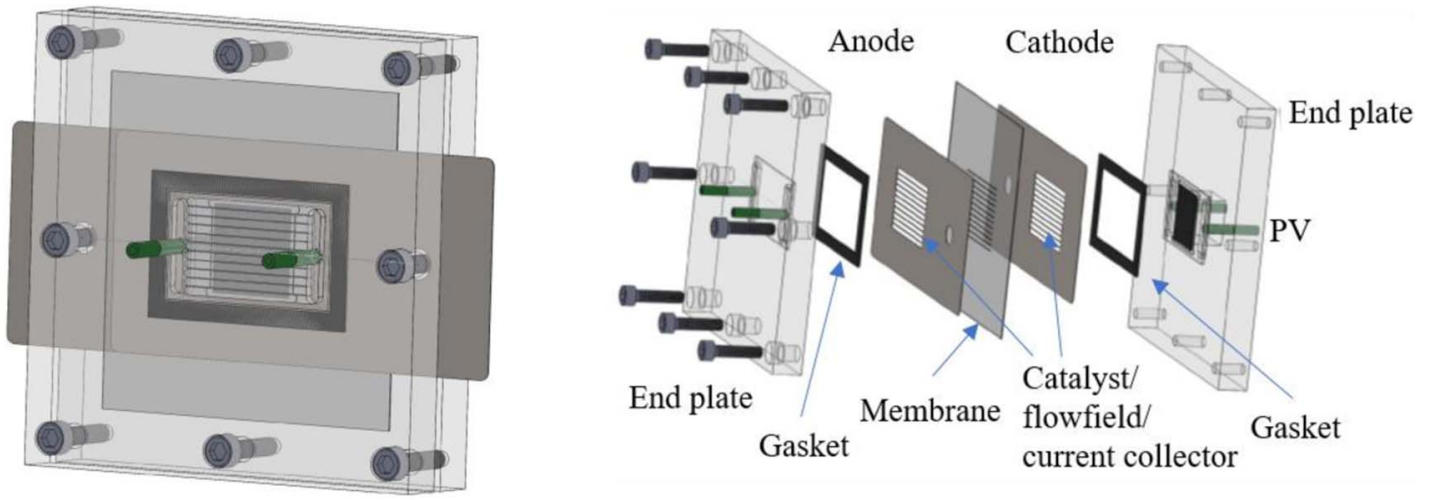

(a)
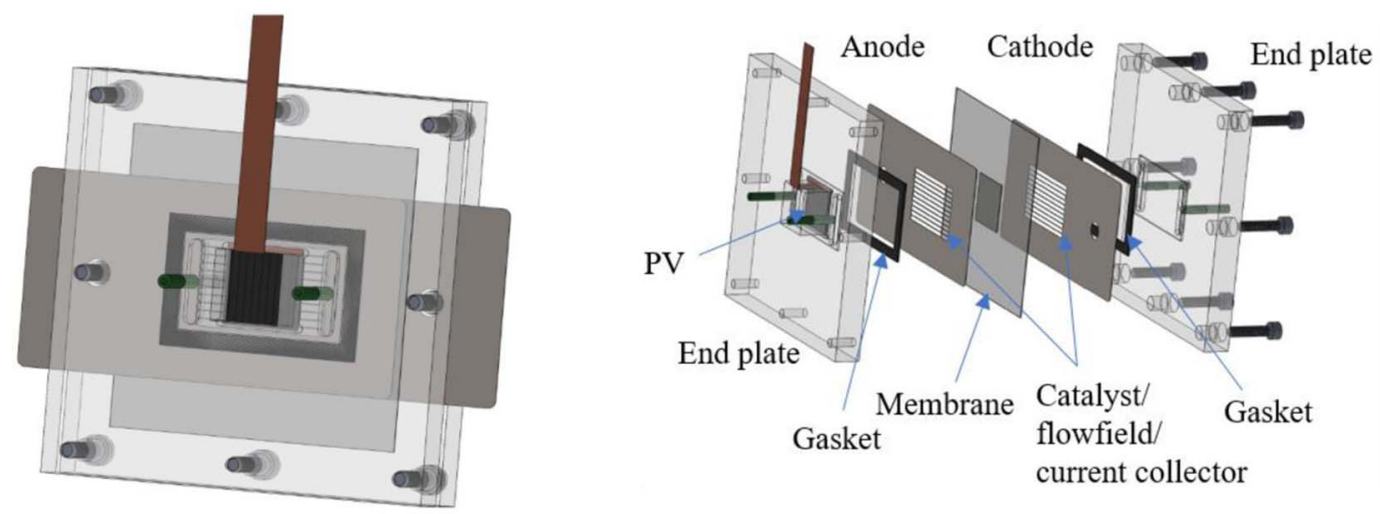

(b)

Figure 1. Vapor (liquid) PEC test bed in two different configurations: a) PV sitting in the cathode compartment (photocathode); b) PV sitting in the anode compartment (photoanode).

non-transparent catalyst layer, thereby providing the maximum possible electrochemical durability and performance. Simultaneously, the maximum possible PV area is exposed to the photon source and unobstructed by even semi-optically transparent cell components. Between the endplates and the tantalum foils, gaskets are used to seal the fluids. For this purpose, a silicon elastomer (BISCO HT-6220, $0.5 \mathrm{~mm}$ thick) or Kalrez (perfluoroelastomer, K\# 5021, Compound 6375, $0.5 \mathrm{~mm}$ thick) is used.

In this work, untreated, $\mathrm{H}^{+}$exchanged Nafion with a thickness of $127 \mu \mathrm{m}$ (Nafion 115) or $27.5 \mu \mathrm{m}$ (Nafion XL) were used as the PEM. The membranes were used as received, and assembled dry in order to minimize gas crossover during operation. Pt and Ir were used as electrocatalysts for the hydrogen evolution (HER) and oxygen evolution (OER) reactions, respectively. These electrocatalysts were deposited by radio frequency (RF) sputtering onto the respective cathode and anode sides of the membrane and Ta foil flowfields. The Ta foils were solvent-cleaned by sequential sonication in acetone (BDH, semiconductor grade) and isopropanol (BDH, semiconductor grade), followed by rinsing with deionized water and drying under high purity $\mathrm{N}_{2}$. Prior to sputter deposition, the Ta foils were etched in a mixture of concentrated nitric and hydrofluoric acid in order to increase the active surface area and improve the adhesion between the catalyst and substrate. The as-etched flowfields were again rinsed with DI water, dried under $\mathrm{N}_{2}$, and then immediately loaded into an AJA International 5-gun magnetron sputtering system for catalyst deposition. The chamber was equipped with $\mathrm{Pt}(99.99 \%)$ and Ir $(99.9 \%)$ targets made in-house at Lawrence Berkeley National Laboratory. Prior to sputter deposition, the substrates were cleaned by plasma etching at $15 \mathrm{~W}$ for $5 \mathrm{~min}$ in an Ar plasma at 30 mTorr. Immediately after this treatment, a $100 \mathrm{~nm}$ layer of catalyst was deposited by RF sputtering in a 3 mTorr atmosphere of Ar, at $150 \mathrm{~W}$. A Ti mask was used to allow for sputtering of catalysts on the membrane that overlap with the lands of the flowfield. The resulting thickness of deposited $\mathrm{Ir}$ and $\mathrm{Pt}$ was $100 \mathrm{~nm}$, controlled by monitoring the sputter deposition rate with a quartz crystal. After deposition, the thickness was confirmed with a profilometer. The total amount of deposited catalyst per electrode corresponds to a loading of $0.2 \mathrm{mg} \mathrm{cm}^{-2}$, and a total PGM loading of $0.4 \mathrm{mg} \mathrm{cm}^{-2}$, which is one order of magnitude lower than a conventional PEM electrolyzer MEA corresponding to $3-5 \mathrm{mg} \mathrm{cm}^{-2} \cdot 3,22$

Photovoltaic.-Commercially available triple-junction GaAs solar cells (Type: TJ Solar Cell 3T34C) from AZUR SPACE Solar Power $\mathrm{GmbH}$ with $\mathrm{InGaP} / \mathrm{GaAs} / \mathrm{Ge}$ sub-cells on a Ge substrate were used to drive the electrolyzer or PEC cell, the electrical data for that solar cell is available on the manufacturer's website. ${ }^{23}$ The as-received cells (active area $30.18 \mathrm{~cm}^{2}$ ) were diced into $1 \times 1 \mathrm{~cm}^{2}$ pieces to fit into the endplates of the PEC. After dicing, the material was cleaned in a heated xylene bath at $50^{\circ} \mathrm{C}$, rinsed with isopropanol, then dried under flowing high-purity $\mathrm{N}_{2}$. No edge passivation was applied, which led to some reduction of power conversion efficiency relative to the as-received cell. Next, the PV was mounted into the PMMA chassis (Figure 2) in which the PV can be electrically wired to an electrolysis cell to form a PV-E, or integrated into the electrolysis cell in direct contact with the cathode establishing a solution junction with the Nafion electrolyte for PEC operation. In either case, the PV was mounted into a recessed ledge in the PMMA and secured on the back side along the edges of the PV using the same EPO-TEK epoxy mentioned above (see Figure 1 and Figure 2). For photocathode applications, a wire was attached 

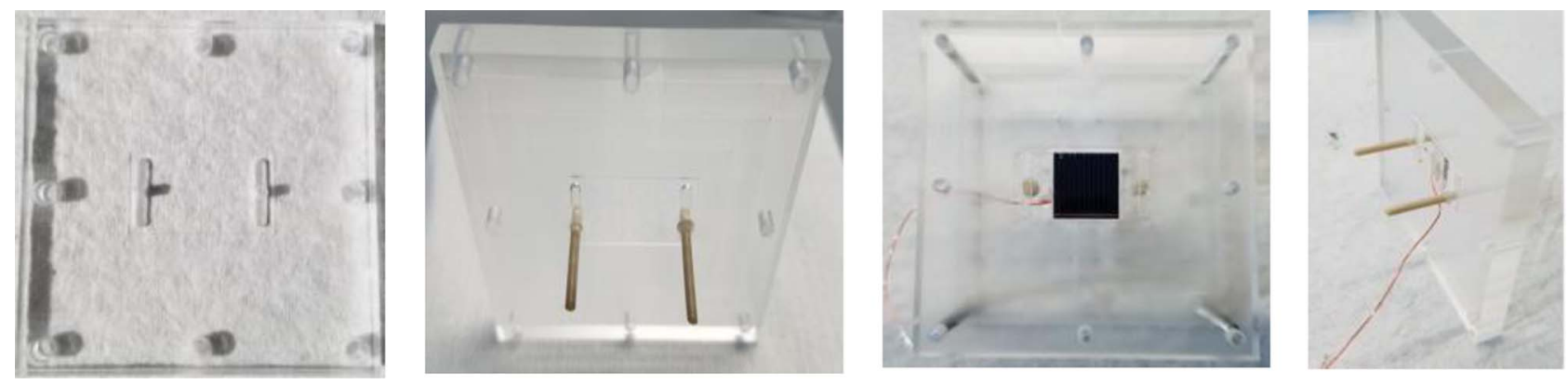

Figure 2. Test bed components (from left to right): Transparent endplate; with tubes (beige) added; endplate with PV attached; PV endplate from the back side showing wire connection and tubing.

with $\mathrm{Ag}$ conductive glue to the back of the PV for current collection and is secured with more epoxy, while the front of the PV was in direct contact with the cathode tantalum flowfield. For photoanode operation, a copper strip was used to make contact along the edge of the front of the PV for current collection, while the back side is sputtered with gold as a protective layer and Ir as catalyst and makes contact with the anode tantalum flowfield.

Cell assembly.-A mounting plate is used to assemble the components; the plate consists of a holder with four screws that protrude up and fit into the holes of the backplate. First, the backplate is placed on top of the mounting table, which allows the entire cell to be aligned easily as the layers are stacked. Then, the cathode gasket is centered on the back endplate, followed by the cathode tantalum foil. In the case of the PEC photocathode design, the electrical stripes of the photovoltaic front side should be perpendicular to the wires of the tantalum foil to improve the contact. The stripes of the catalyst on the membrane have to be aligned well with the wires of the tantalum foil flowfield to establish good electrical contact, and to minimize the percentage of light that gets blocked coming from the side of the front endplate going to the photovoltaic embedded in the back endplate. Next, the anode gasket is positioned on top of the anode tantalum foil, followed by the front endplate. The cell is then compressed by tightening the eight screws evenly and in a star-shaped pattern to a final torque of $0.1 \mathrm{Nm}$. The gasket's thickness and endplate load is tuned in order to provide adequate active area pressure but not to damage the membrane by the lands of the tantalum flowfields, confirmed with pressure film. ${ }^{24}$ However, we emphasize that without the use of any gas-diffusion layers or porous-transport layers, whose opacity precludes their application, areas of the membrane are unsupported and thus affected by pressure differences. The assembled cell as either PV-E or PEC can be seen in Figure 3.

Device performance measurements .- All electrochemical, photoelectrochemical, and solid-state photovoltaic data were collected using a Biologic SP-300 potentiostat. Dark (without illumination, potentiostat biased), two-electrode measurements were taken using counter electrode and reference electrode leads connected to the cath-
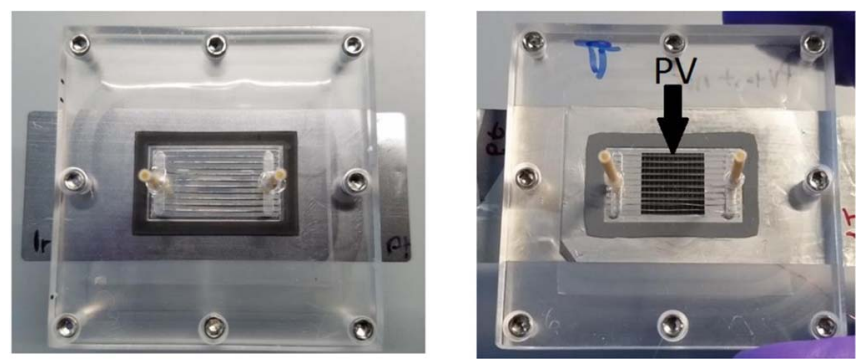

Figure 3. Test bed as PV-E, with PV as a separate chassis out of view (left) and PEC with integrated electrolyzer and PV (right). ode, while the working electrode was connected to the anode. For on-sun experiments (with illumination, potentiostat un-biased) in either PV-E or PEC mode, the reference and counter electrodes were connected to the cathode and front contact of the PV, while the working electrode was connected to the anode and the back contact of the $\mathrm{PV}$ as the potentiostat served to measure the current flowing through the cell. Simulated sunlight was produced using an AAA-rated Newport Oriel Sol3A equipped with a Xe lamp and AM $1.5 \mathrm{G}$ filter to obtain a $12^{\prime \prime} \times 12^{\prime \prime}$ illumination area. A Newport $91150 \mathrm{~V}$ reference cell with a monocrystalline silicon solar cell and an integrated thermocouple was used to measure the solar irradiance. The reference cell was calibrated and traceable to both NREL and to the International System of Units (SI). Solar water-splitting devices were positioned to receive 1 sun $\left(1000 \mathrm{~W} \mathrm{~m}^{-2}\right)$ illumination to the $\mathrm{PV}$ front surface, as determined using the reference cell.

The produced gases were analyzed using a gas chromatograph (GC, Agilent 7890A, Santa Clara, CA). The gas chromatograph was equipped with two channels, between which measurements could be toggled. The cycle time for each run was $7.5 \mathrm{~min}$. Calibration curves for $\mathrm{H}_{2}$ and $\mathrm{O}_{2}$ were obtained with a 3-point calibration curve at 100,1000, $9918 \mathrm{ppm}$ and 100, 1000, $8000 \mathrm{ppm}$, respectively. Prior to calibration, the system was purged for $20 \mathrm{~min}$ with $10 \mathrm{sccm}$ of the calibration gas of interest, after which the mass-flow controllers (Alicat Scientific, $10 \mathrm{sccm}$ full scale) were set to the desired flow rate. A minimum of ten samples was collected for each calibration point. On the cathode side, a dry $\mathrm{N}_{2}$ sweep gas was used flowing through the cathode compartment of the cell into a hydrogen/vapor water separator; the hydrogen gas went out of the headspace and into the GC. On the anode side, Milli-Q water (MilliporeSigma, Burlington, MA, resistivity of $>18.2 \mathrm{M} \Omega^{*} \mathrm{~cm}$ ) was pumped through the cell and into the oxygen/liquid water separator; the head space of the separator was purged with dry $\mathrm{N}_{2}$ into the GC for analysis. Two syringe pumps with a Dual Pump Plumbing Kit (New Era Pump Systems Inc., Farmingdale, NY) were used to maintain continuous flow of water at a rate of $0.05 \mathrm{ml} \mathrm{min} \mathrm{m}^{-1}$. The flow rate of purge gases was controlled using mass flow controllers, a combination of $0.5 \mathrm{sccm}$ for the anode and $10 \mathrm{sccm}$ for the cathode showed the best performance in the case of a liquid-feed anode. In the case of vapor-feed tests, humidified nitrogen flowed into either the anode and cathode compartments or only the anode compartment, at rates between 25 to $50 \mathrm{sccm}$. A test stand (Fuel Cell Technologies. Inc., Albuquerque, NM), equipped with a heated humidifier filled with Milli-Q water and heated gas lines, was used to humidify the feed gases. To ensure minimal water condensation, the gas line temperature was set to $50^{\circ} \mathrm{C}$, which is $5^{\circ} \mathrm{C}$ higher than the humidifier and cell temperature after exposure to the light source for at least 30 minutes. This results in a relative humidity of roughly $100 \%$ in the electrode compartment(s) fed by humidified $\mathrm{N}_{2}$.

\section{Results and Discussion}

We systematically evaluated the impact of materials of cell or MEA manufacture, cell preparation, and operating conditions (liquid and vapor feed), with the goal of achieving stable electrolysis and 


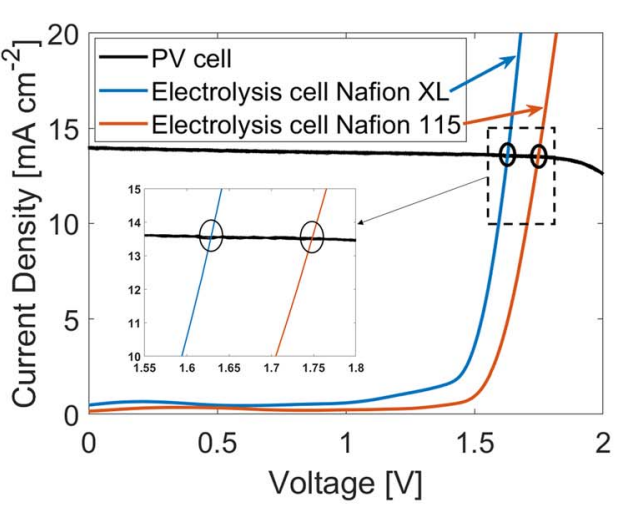

(a)

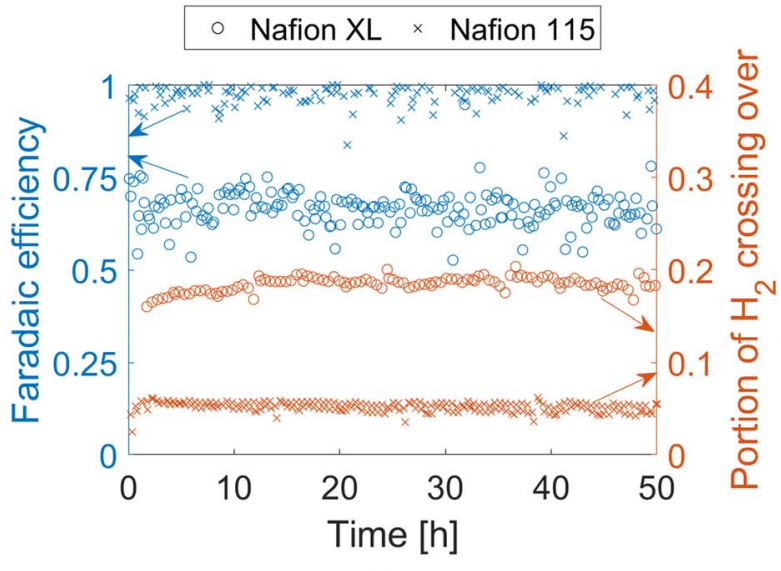

(b)

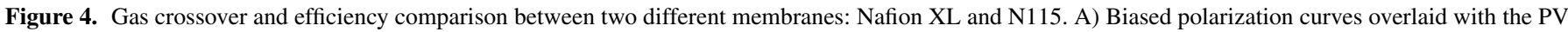

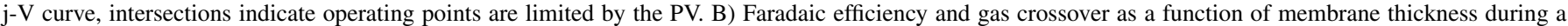
50-hour PV-E durability test with an average current of $10.5 \mathrm{~mA} \mathrm{~cm}{ }^{-2}$ during both experiments.

PV-E conditions in either mode of operation (liquid water or water vapor) prior to PEC testing. The results of these tests are provided in the Supplemental Information.

Effect of membrane thickness.-To maximize the amount of light that reaches the PV sitting in the cathode compartment, the membrane needs to be as thin as possible. A thinner membrane also results in lower ohmic resistance, however at the expense of increased hydrogen crossover. ${ }^{25,26}$ Thus, we evaluated two membrane thicknesses of the perfluorosulfonic-acid chemistry. The thinner membrane Nafion XL (27.5 $\mu \mathrm{m}$ thick) also contained a PTFE membrane support material, while the thicker membrane was Nafion 115 (127 $\mu \mathrm{m}$ thick). As expected, decreasing the membrane thickness improved the electrolysis performance by $120 \mathrm{mV}$ at the operating current density due to the lower ohmic resistance (Figure 4a). However, this resulted in a significantly increased hydrogen crossover (Figure $4 \mathrm{~b}$ ) from $6 \%$ to $18 \%$, which reduces the solar to hydrogen (STH, Equation 1) efficiency:

$$
\mathrm{STH}=\frac{\text { current density } \times 1.229 \mathrm{~V} \times \text { faradaic efficiency }}{\text { illumination power density }}
$$

We note that crossover hydrogen from the cathode to the anode, effectively reduces the amount of produced $\mathrm{H}_{2}$. This is reflected in the faradaic efficiency which is the difference between the amount of $\mathrm{H}_{2}$ produced from the supplied current and the amount of $\mathrm{H}_{2}$ that does not go into the cathode product stream. Thus, the resulting faradaic efficiency of over $90 \%$ using Nafion 115 decreases to about $70 \%$ using Nafion XL (see Figure 4b). Since the intersection with the PV power curve limits the operating point of the cell, as shown by the intersection points of the photocurrent with the OER current during electrolysis (Figure 4a), maximum achievable PV-E or PEC current density is controlled by the PV, regardless of the thickness of membrane used. However, the crossover is severely affected as shown by the $\mathrm{H}_{2}$ crossover and faradaic efficiency in Figure 4b. Hence, Nafion 115 was chosen for further studies in this work. As shown in Figure $4 \mathrm{a}$, a maximum current density of at least $20 \mathrm{~mA} \mathrm{~cm}^{-2}$ below $2 \mathrm{~V}$ is achieved in biased electrolyzer experiments with the MEA. This is well above the short circuit current of the PV which represents the upper limit of current that it can supply to the MEA (as described in the SI, Figure S1). Thus, for the PV-E and PEC experiments, the intersections in Figure 4a between the electrolyzer and PV represent the maximum photocurrent density of nearly $14 \mathrm{~mA} \mathrm{~cm}^{-2}$ achievable during operation. We measured the transmission of light through N115 and found it to transmit $97 \%$ of light across the visible spectrum. Therefore, it is not expected that the membrane is limiting under PEC conditions. However, the choice of liquid water pump circulation and delivery pump could limit the durability of the PEC cell due to im- proper membrane hydration conditions, as presented in the SI (Figure S2 and Figure S3).

Liquid-water feed PV-E and PEC.-Initial integration utilized the PV-E setup, wherein the PV connects to the anode or the cathode side of the cell, but still not fully integrated (see Figure 3 left). In both anode and cathode configuration, PV area $\left(1 \mathrm{~cm}^{2}\right)$ and catalyst areas $\left(1 \mathrm{~cm}^{2}\right)$ are the same, only the geometry differs. In the cathode PV-E configuration, the front of the PV was contacted by a tantalum foil and wired to the electrolysis cell, in the anode, the electrical contact between the back of the PV and the anode compartment was established by conductive glue.

First, to evaluate the long-term durability of the electrolysis MEA and the PV contact, a photocathode PV-E configuration was used with a syringe pump setup, and Nafion 115 . Under these conditions, a maximum solar to hydrogen (STH) efficiency above $12 \%$ was observed with stable performance (STH efficiency loss of less than $1 \%$ ) for more than 100 hours (Figure 5b, photocathode). Also, the expected $\mathrm{H}_{2} / \mathrm{O}_{2}$ stoichiometry of 2 was observed (see Figure S4a), indicating high faradaic efficiency (Figure S4b), and the stability of the MEA was confirmed by cyclic voltammetry measurements before and after the long-term operation (Figure S4c).

Second, the photoanode configuration was tested keeping all the other conditions the same. The initial electrolysis performance of the photoanode cell, showed in Figure 5a, is lower than the photocathode performance. This was probably due to the use of dense and thick sputtered catalyst layers on the membrane, which lack the porosity to produce superior electrocatalytic performance. ${ }^{27}$ A more reasonable approach is to utilize a conventional coating process for the catalyst layer which no longer has the requirement to be optically transparent. On the other hand, the PV performance is more efficient in the photoanode configuration because of the improved front contact to the PV (compare with Figure S1). However, in both cases, the achievable electrical current of the whole system is still limited by the maximum current available from the PV, see intersections 1 and 2 in Figure 5a. Both configurations show similar initial STH efficiencies and insignificant degradation rates which results in over $12 \%$ STH efficiency after more than 100 hours, see Figure $5 \mathrm{~b}$.

For PEC operation, the PV was glued into the cathode compartment endplate of the electrolysis cell and put directly in contact with the cathode catalyst and electrolyte to form an integrated PEC cell. The anode was fed with liquid water from the dual syringe pump while the cathode was flushed with dry nitrogen and light was provided by the solar simulator through the cell. Initial STH was around 7\%, which is $42 \%$ lower than the PV-E configuration as a result of the reduced light transparency to the PV. More significantly and unexpectedly, the STH 


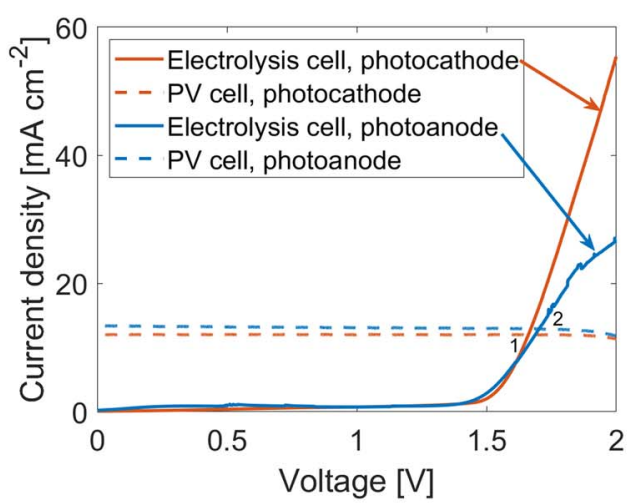

(a)

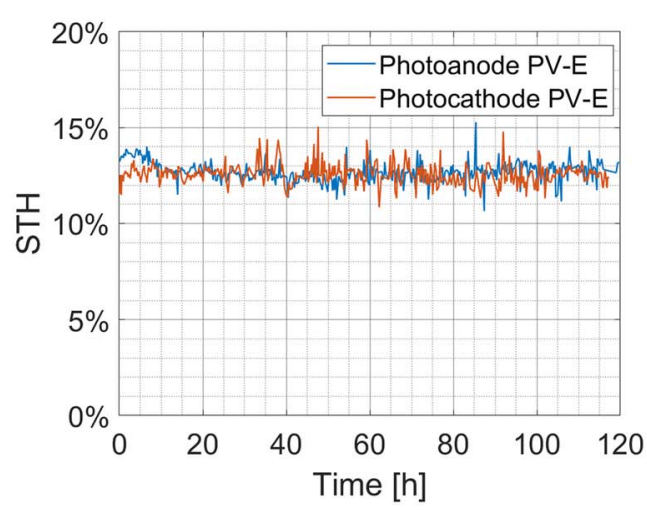

(b)

Figure 5. Comparison between photoanode and photocathode operation in PV-E configuration, liquid feed to the anode using double syringe pump setup (a) Biased polarization curves for photoanode and photocathode MEAs overlaid with PV cell performance showing the operating performance of either cell is not limited by the MEA but by the PV. (b) Long-term durability test of the unbiased PV-E cells showing equivalent durability and STH in both photoanode and photocathode configurations culminating in more than $12 \%$ STH efficiency for over 100 hours continuous operation, using a liquid-water fed double syringe pump setup.

and operating current decreased rapidly with time and after 24 hours the efficiency was too low to continue the experiment (see Figure 6a). This accelerated degradation is due to pitting corrosion of the PV (see Figure 6 b) from liquid water that diffused or was electro-osmotically transported from the anode to the cathode. The resulting corrosion drastically affected the PV efficiency, see Figure $6 \mathrm{c}$ for comparison before and after PEC test. The open-circuit voltage of the PV as well as the fill factor dropped significantly, hence making it impossible for the PV to provide enough potential to drive water splitting. Since the material properties of the sub-cells in the triple junction PV and the antireflection coating (ARC) are carefully matched ${ }^{28}$ it is important to protect each layer of the PV from damage. If one of the sub-cells or the ARC falls victim to corrosion or mechanical damage, the light amount and distribution to the sub-cells changes, which results in a current drop and mismatch and therefore decrease in PV efficiency. For this reason, either the PV front surface needs to be protected for efficient PV operation in the cathode compartment or the amount of water reaching the PV needs to be reduced substantially. ${ }^{29,30}$

Vapor feed PV-E and PEC.-The same MEA setup used in liquid water fed PV-E mode was used for evaluating performance and durability of vapor-feed operation. For vapor-feed operation, the syringe pump is replaced by nitrogen gas with a controlled water content ( $\sim 100 \%$ relative humidity) by using mass-flow controllers as described above. Initially, humidified gas was supplied to both sides of the cell (dual vapor feed). Comparing the liquid-water-feed versus vapor-feed performance (Figure 7a), the performance of the dual vapor-feed cell during the limited timeframe for this potentiodynamic, biased test was not impacted by either the lower mass transport of vapor versus liquid, nor the reduced conductivity of the membrane under vapor conditions. Furthermore, the same STH efficiency of $12 \%$ was achieved (Figure 7b) compared to liquid feed PV-E operation, and the durability was also unaffected as the STH remained above $11 \%$ after 100 hours, indicating that the cell operation was once again durable in dual vapor-feed mode.

Since water on the cathode side of the cell is detrimental to the $\mathrm{PV}$, vapor-feed operation may reduce the corrosion by decreasing the amount of liquid water in the cell without impacting the electrochemical performance significantly. However, we found that using the same PEC configuration and with humidified feeds in both compartments results in similar degradation. Thus, in order to decrease degradation in PEC operation, a more drastic reduction in water content in the cathode compartment must be achieved. To accomplish this, water vapor is only supplied to the anode side of the cell while the cathode is flushed with dry nitrogen gas. In this configuration, the PEC's durability increased to over 4 days while still achieving the same peak STH of $\sim 7 \%$ (Figure $8 \mathrm{a}$ ) as the liquid-water fed PEC. However, in this configuration, the cell is very sensitive to gas flow conditions and humidity. During the first 22 hours of operation (part I), the bubble humidifier was heated to $50^{\circ} \mathrm{C}$, which is slightly

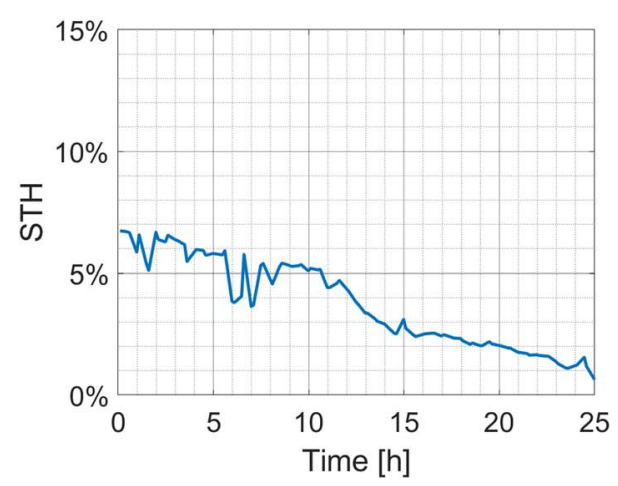

(a)

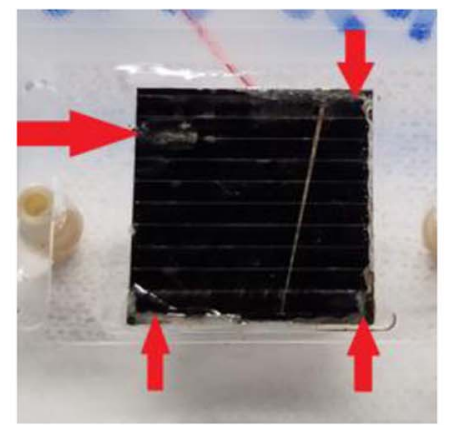

(b)

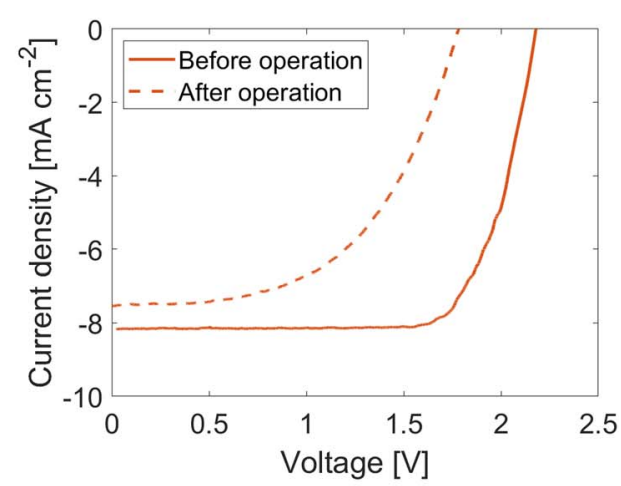

(c)

Figure 6. Corrosion arising on the PV front surface during unprotected PEC photocathode operation with liquid water fed to the anode (a) Decreasing STH over time due to PV corrosion; (b) Optical image showing corrosion on PV surface after cell disassembly; (c) Comparison of PV efficiency from beginning of life to after 25 hours of durability testing showing decrease in fill factor and electrical current. 


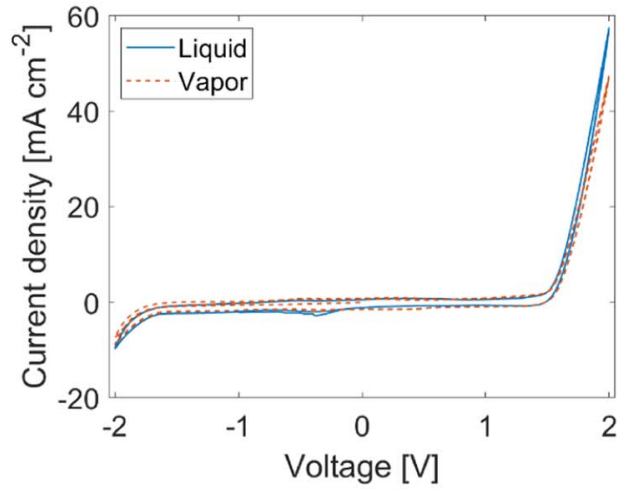

(a)

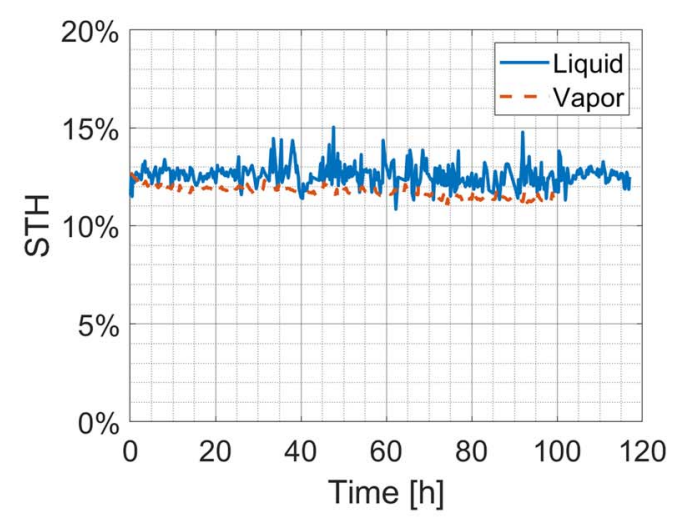

(b)

Figure 7. Comparing PV-E performance in photocathode configuration between liquid-water-anode and dual (anode and cathode) vapor feed (a) Biased polarization curves for liquid water and vapor feed. (b) Long-term durability test of the unbiased PV-E cells showing equivalent durability and STH in both liquid-water and vapor feed culminating in more than $11 \%$ STH efficiency for over 100 hours continuous operation.

above the cell temperature $\left(45^{\circ} \mathrm{C}\right)$ in order to ensure $100 \%$ relative humidity in the anode compartment. At these conditions, the membrane in the PEC periodically dried and re-humidified, resulting in a temporary increase in membrane resistance and fewer water molecules being available for the water-splitting reaction. In other words, the cell operated under water mass-transport limiting conditions, which also limited the amount of water that made it to the cathode side of the cell, thus extending the lifetime of the PV. However, this resulted in fluctuation of STH efficiency between around 5 and $8 \%$ as the cell performance oscillated. To balance the cell's water supply and water consumption, during the next 20 hours ( 22 to 42, part II in Figure 8a) the temperature of the humidifier was increased to $60^{\circ} \mathrm{C}$, resulting in super-saturation and some liquid water in the anode compartment. This decreased the dry time of the cell in which the membrane was being re-humidified and resulted in a higher average STH efficiency over time and decreased frequency of oscillation. Then, after 42 hours of operation (part III), the anode gas flow rate was doubled from an initial $25 \mathrm{sccm}$ to $50 \mathrm{sccm}$ to deliver even more water to the PEC. As a consequence, the membrane did not dry out and the fluctuations in STH efficiency were entirely removed. Initially, this can be seen as a benefit due to improved steady-state performance, however, after around 10 hours of operation under those conditions, the STH efficiency gradually decreased, mimicking the failure of the liquid-water
PEC test. Under these conditions, there is an excess of water in the $\mathrm{PEC}$, resulting in slow corrosion of the PV. The performance before and after operation of the two parts, PV and electrolysis cell, is shown separately in Figure 8b. The open-circuit voltage, short-circuit current and fill factor of the PV dropped considerably, reducing its ability to drive reactions in the electrolysis cell. While the decrease in electrolytic activity would not influence the resulting current density of the overall device using a new PV (compare intersections labeled 1 and 2 in Figure $8 b$ ), it further drops the current density in combination with the degraded PV because of the PV's decreased fill factor (compare intersections labeled 3 and 4). Since the water balance is critical to long term operation of the PEC, it is essential to prevent or block liquid-water accumulation and condensation at the PV, even though it might cause more oscillatory behavior. We also evaluated the operation of the vapor fed PEC cell under more aggressive diurnal cycling which is included in the SI (Figure S5).

After disassembling the PEC, the PV was examined using SEM/EDX and XRF. The corrosion of the PV was obvious visually, in the form of pits. The XRF, SEM/EDX measurements (Figure 9) show that the Ag/Au electrical contact was largely intact, except in the locations where pits where formed. The corrosion starts with the $\mathrm{Al}_{2} \mathrm{O}_{3}$ and $\mathrm{TiO}_{\mathrm{x}}$ anti-reflective coating layer, and then proceeds to the top layers (InGaP and GaAs) of the triple-junction PV. The patterns

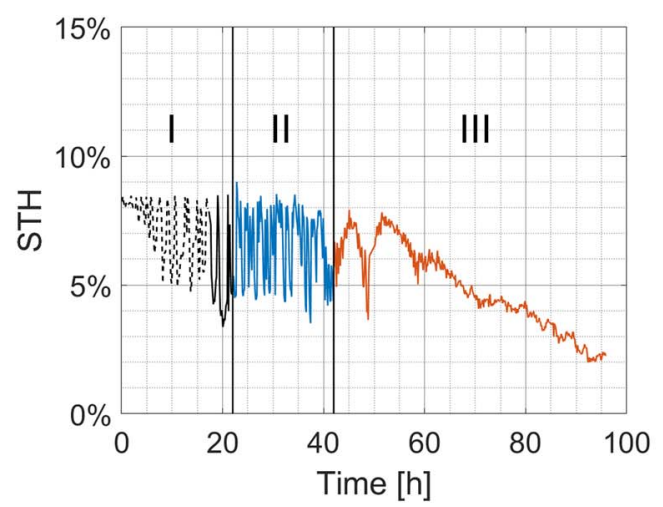

(a)

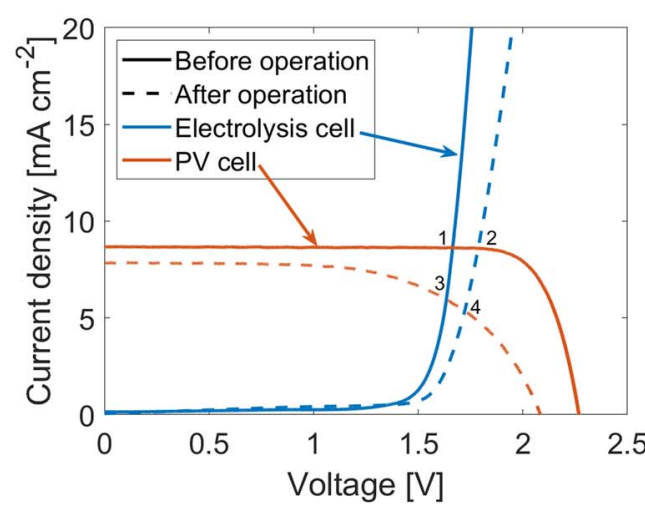

(b)

Figure 8. PEC photocathode operation with water vapor fed to the anode and dry $\mathrm{N}_{2}$ to the cathode. (a) STH efficiency plotted as a function of time during the durability test lasting more than four days. Sections demark different operating conditions in order to manage the cell water consumption and hydration. I) $100 \%$ RH condition resulting in oscillating STH due to membrane dehydration; II) oversaturated condition with decreased oscillation periods; III) increased flow rate further increasing the membrane hydration but causing liquid water to collect on the cathode and corrode the PV. Note for the first 17 hours (dashed line), direct gas detection was not available, the STH efficiency was calculated assuming the same faradaic efficiency as in the first 10 hours of direct gas detection with a gas chromatograph (solid line). (b) MEA CV overlays with PV j-V curves before and after testing, showing a combination of electrochemical degradation and PV corrosion resulting in decreased STH after 96 hours. 
(a)

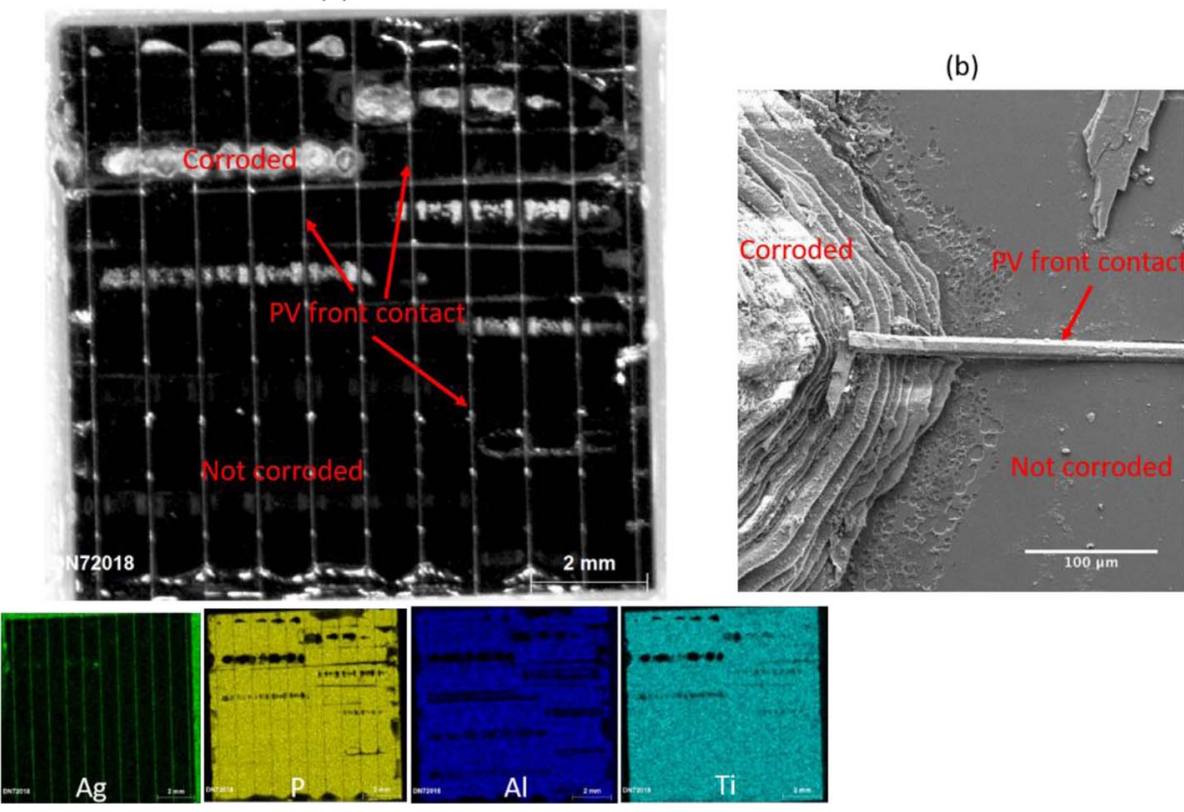

(c)

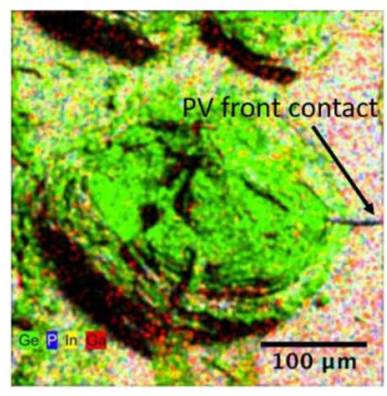

Figure 9. Failure analysis characterization of the PV after durability testing. a) XRF with insets b) SEM c) EDX pictures of the corroded PV after 100 hours. Corrosion only occurs on some parts of the PV while other parts remain unaffected as seen in the XRF and SEM images. The Au/Ag front electrical contacts are largely intact except near corroded spots. The $\mathrm{Al}$ and Ti oxide top layers and InGaP semiconducting layers are only affected in certain areas that visually correspond to the channels that are formed by the Ta flowfields. The corrosion forms surfaces similar to impact craters: the thickness of the PV at the center of a crater is reduced, only the germanium substrate of the triple-junction PV is left, while at the edge of the crater the thickness of the PV increased, which can cause the front contact to lift and break.

of corrosion follow the Ta flowfields of the device, the corrosion of the semiconducting layers which are gradually removed in some spots during the operation inevitably lead to the decrease in PV's performance. Visual analysis of the video feed (see Supplemental video) during PEC operation clearly shows disparity between liquid flooded areas, which indicates that the main cause of corrosion of the PV is condensed water that accumulates locally and causes degradation. At this time, we do not want to speculate as to the mechanism of corrosion through the $\mathrm{Al}_{2} \mathrm{O}_{3}$ and $\mathrm{TiO}_{\mathrm{x}}$ layers, except that it most likely has to do with the following: The PV is in contact with the Nafion membrane (which is strongly acidic) through the channels of the flowfield (depending on the balance of fluid pressure between the anode and cathode); the water emerging through the membrane from the anode to the cathode is protonated which renders it more acidic than DI water. XRF analysis of the MEA and cathode Tantalum foil indicate that the sputtered Pt and Ir catalyst layers are largely preserved during operation (Figure S6).

Barrier layer operation.-Finally, having characterized the nature and operation of the integrated MEA device, we introduced a barrier layer to the PEC to improve its durability by further limiting access of water to the PV. The approach used is derived from a previous publication, ${ }^{15}$ we first ensured adequate contact between the PV and the Ta flowfield, and then coated the exposed PV surface with epoxy (EPO-TEK 302-3M). The cell with barrier coating was first evaluated under steady state operation with 1 sun illumination intensity and anode vapor feed (Figure 10a), to compare it with the most stable PEC operating condition without barrier layer (Figure 8). While the oscillations due to membrane dehydration persist, the STH is steady over 100 hours of testing, peaking at $7.5 \%$. Due to the ruggedness

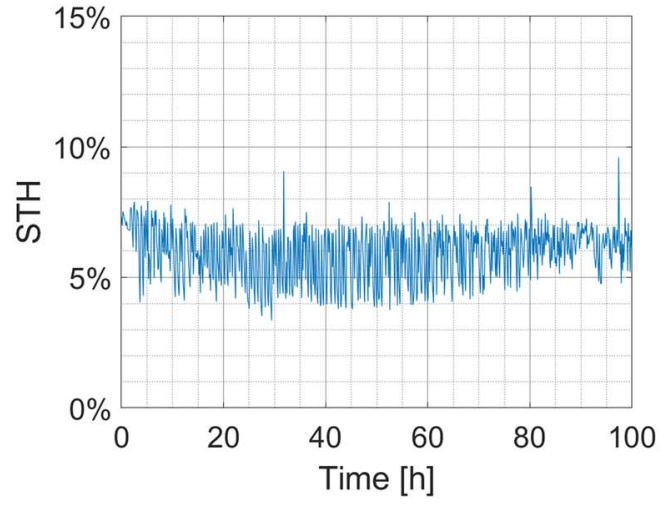

(a)

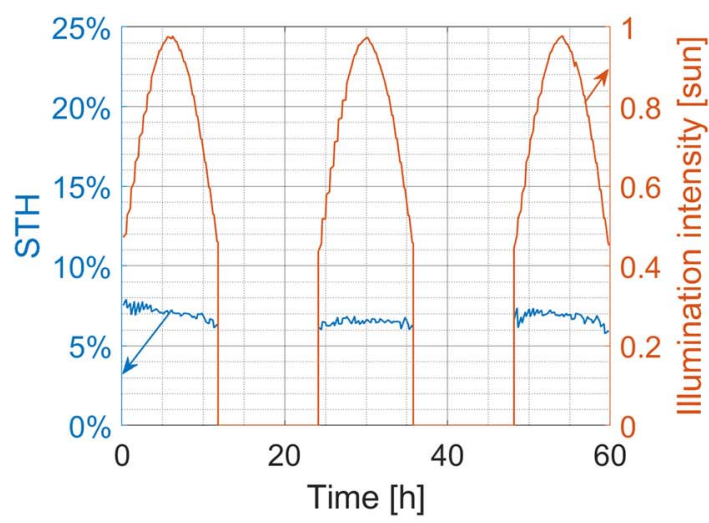

(b)

Figure 10. Cell operation with vapor feed and a barrier layer. (a) First 100 hours of durability test with anode vapor feed only, STH efficiency plotted as a function of time shows durable performance with fluctuations due to hydration/dehydration cycles of the membrane. (b) Diurnal cycling performed with dual vapor feed after 122 hours of steady state testing at 1 sun. The STH output was largely not affected during the cycles which indicated the durability of the cell. 


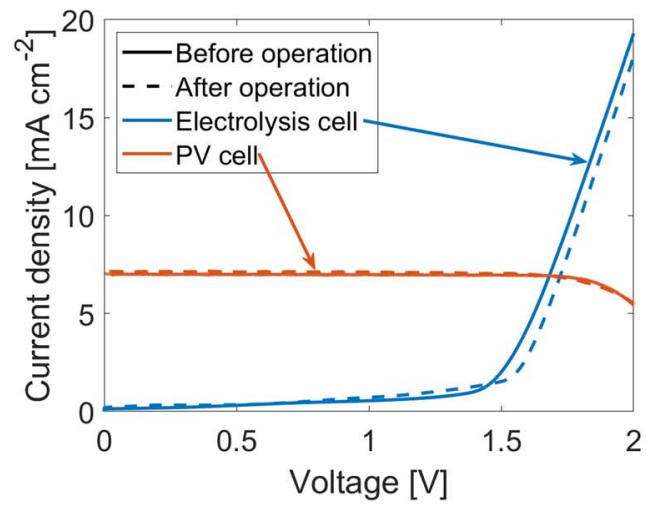

(a)

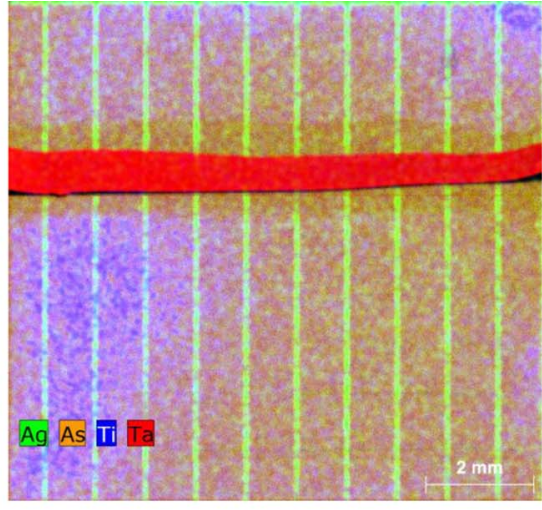

(b)

Figure 11. Vapor fed device with barrier layer characterization. a) MEA CV overlays with PV j-V curves before and after testing, showing unchanged PV operation and slight electrochemical degradation of the cell after a cumulative 122 hours of steady state durability testing and three diurnal cycles. b) XRF measurement of $\mathrm{PV}$ after testing, showing the PV surface is intact with neither signs of pitting nor loss of antireflection coating/semiconducting components. Note that the tantalum strip shown is from the altered current collecting scheme used with the barrier coating.

of the barrier coating, we subsequently used dual vapor flow on the same sample for an additional 22 hours of further steady state operation (Figure S7a), which decreased the amplitude of oscillations. To explore more realistic operation, diurnal cycle testing was conducted using the dual vapor-feed (Figure 10b), $\mathrm{j}-\mathrm{V}$ curves of the PV at different light intensities can be seen in Figure S8a. Diurnal cycling is the most realistic test condition for a PEC cell as it mimics the natural day and night transitions and causes additional stresses to the PEC. This also provides an understanding of the effective turndown ratio of the integrated cell. The results of the diurnal cycling again showed stable operation and a peak STH of $7.5 \%$, while for an unprotected PV the STH dropped from $7.5 \%$ to around $2.5 \%$ over the course of the diurnal test (Figure S5a).

Post testing analysis of the unprotected PEC device revealed that the degradation in performance was primarily due to degradation of the PV (CV and j-V curves in Figure S5b). In contrast, post testing analysis of the barrier protected device showed that the cell was largely unaffected after a cumulative 182 hours of testing (122 hours of steady state followed by three diurnal cycles). First, j-V curves obtained before and after operation show that the PV performance was unaffected, while CVs of the MEA showed a slight degradation of $\sim 50 \mathrm{mV}$ (Figure 11a). A comparison of the peak current densities at 1 sun across the steady state and diurnal tests (Figure S7a and b, Figure $\mathrm{S} 8 \mathrm{~b}$ ) also show that the electrical current did not degrade over the 182 hours. Furthermore, we analyzed the PV surface after testing with $\mathrm{XRF}$ which showed minimal to zero loss of functional components as a result of cell operation (Figure 11b).

\section{Summary}

Photoelectrochemical cells have long been tested in liquid electrolyte, where they suffer from short durability due to exposure of the PV to the liquid electrolyte. The membrane-electrode-assembly (MEA) style PEC cell presented herein is a deviation from that paradigm as a solid electrolyte is used with a vapor feed which reduces the amount of liquid and electrolyte contact with the PV, therefore offering the ability to prolong the life of the PEC. While in this study commercial III-V semiconductors were used in order to optimize, characterize and demonstrate the testbed itself, any PEC photoanode, or photocathode or both could be used if they are transparent. We demonstrate the durability of the electrochemical components which under liquid and vapor conditions in PV-E mode resulted in a STH efficiency of $12 \%$ and a durability of at least 100 hours using loadings that are lower compared with a commercial PEM electrolyzer based PV-E system ( $0.4 \mathrm{mg} \mathrm{cm}^{-2}$ vs. $3-5 \mathrm{mg} \mathrm{cm}^{-2}$ respectively) which would operate at a STH efficiency of $16 \%$ under the same conditions.
Under PEC operating conditions with either liquid or vapor feed operation the STH efficiency is reduced to below $7.5 \%$ due to obstruction of light to the PV through cell components. Durability suffers as well due to exposure of the PEC to water; under bulk liquid and dual (anode and cathode) vapor feed operation the device lifetime was reduced to 24 hours, while under anode-only vapor operation the PEC slowly degraded over 100 hours. We finally demonstrated the use of a barrier coating on the cell which resulted in a stable STH efficiency of $7.5 \%$ during 182 hours of combined steady state and diurnal testing without any sign of PV degradation. Important conclusions of this study included our findings that PV exposure to water must be minimized, and that minimizing water drag between the anode and cathode could be achieved by operating the device in mass transport-limited regimes.

\section{Acknowledgments}

The authors gratefully acknowledge research support from both: Joint Center for Artificial Photosynthesis (JCAP), a DOE Energy Innovation Hub, supported through the Office of Science of the U. S. Department of Energy under Award Number DE-SC0004993 and the HydroGEN Advanced Water Splitting Materials Consortium, established as part of the Energy Materials Network under the U. S. Department of Energy, Office of Energy Efficiency and Renewable Energy, Fuel Cell Technologies Office, under Contract Number DE-AC0205CH11231. The work performed by JCAP consisted of generating the device conceptualization, proof of concept and catalyst development. The work performed under HydroGEN consisted of device fabrication, optimization, device characterization and failure analysis. Initial laser cutting experiments were supported by COMPRES, the Consortium for Materials Properties Research in Earth Sciences under NSF Cooperative Agreement EAR 11-57758. Authors gratefully acknowledge Frances Houle for guidance, insights and assistance in manuscript preparation.

\section{ORCID}

Tobias A. Kistler (1) https://orcid.org/0000-0001-6458-8024 Adam Z. Weber (1D https://orcid.org/0000-0002-7749-1624

\section{References}

1. S. Almosni, A. Delamarre, Z. Jehl, D. Suchet, L. Cojocaru, M. Giteau, B. Behaghel, A. Julian, C. Ibrahim, L. Tatry, H. Wang, T. Kubo, S. Uchida, H. Segawa, N. Miyashita, R. Tamaki, Y. Shoji, K. Yoshida, N. Ahsan, K. Watanabe, T. Inoue, M. Sugiyama, Y. Nakano, T. Hamamura, T. Toupance, C. Olivier, S. Chambon, 
L. Vignau, C. Geffroy, E. Cloutet, G. Hadziioannou, N. Cavassilas, P. Rale, A. Cattoni, S. Collin, F. Gibelli, M. Paire, L. Lombez, D. Aureau, M. Bouttemy, A. Etcheberry, Y. Okada, and J.-F. Guillemoles, Sci. Technol. Adv. Mater., 19, 336 (2018).

2. M. Gul, Y. Kotak, and T. Muneer, Energy Explor. Exploit., 34, 485 (2016).

3. N. Danilovic, K. E. Ayers, C. Capuano, J. N. Renner, L. Wiles, and M. Pertoso, ECS Trans., 75, 395 (2016).

4. B. Pivovar, N. Rustagi, and S. Satyapal, Electrochem. Soc. Interface, 27, 47 (2018).

5. A. J. Bard and M. A. Fox, Acc. Chem. Res., 28, 141 (1995).

6. B. A. Pinaud, J. D. Benck, L. C. Seitz, A. J. Forman, Z. Chen, T. G. Deutsch, B. D. James, K. N. Baum, G. N. Baum, S. Ardo, H. Wang, E. Miller, and T. F. Jaramillo, Energy Environ. Sci., 6, 1983 (2013).

7. J. Jia, L. C. Seitz, J. D. Benck, Y. Huo, Y. Chen, J. W. D. Ng, T. Bilir, J. S. Harris, and T. F. Jaramillo, Nat. Commun., 7, 13237 (2016).

8. J. W. Ager, M. R. Shaner, K. A. Walczak, I. D. Sharp, and S. Ardo, Energy Environ. Sci., 8, 2811 (2015).

9. M. A. Modestino and S. Haussener, Annu. Rev. Chem. Biomol. Eng., 6, 13 (2015)

10. O. Khaselev and J. A. Turner, Science, 280, 425 (1998)

11. R. C. Kainthla, B. Zelenay, and J. O. Bockris, J. Electrochem. Soc., 134, 84 (1987)

12. M. R. Shaner, K. T. Fountaine, S. Ardo, R. H. Coridan, H. A. Atwater, and N. S. Lewis, Energy Environ. Sci., 7, 779 (2014).

13. C. Liu, J. Tang, H. M. Chen, B. Liu, and P. Yang, Nano Lett., 13, 2989 (2013).

14. H. Mettee, J. W. Otvos, and M. Calvin, Sol. Energy Mater, 4, 443 (1981).

15. K. A. Walczak, G. Segev, D. M. Larson, J. W. Beeman, F. A. Houle, and I. D. Sharp, Adv. Energy Mater., 1602791 (2017).
16. M. M. May, H.-J. Lewerenz, D. Lackner, F. Dimroth, and T. Hannappel, Nat. Commun., 6, 8286 (2015)

17. E. Verlage, S. Hu, R. Liu, R. J. Jones, K. Sun, C. Xiang, N. S. Lewis, and H. A. Atwater, Energy Environ. Sci., 8, 3166 (2015).

18. M. A. Modestino, M. Dumortier, S. M. H. Hashemi, S. Haussener, C. Moser, and D. Psaltis, Lab. Chip, 15, 2287 (2015).

19. C. Xiang, Y. Chen, and N. S. Lewis, Energy Environ. Sci., 6, 3713 (2013)

20. S. Kumari, R. T. White, B. Kumar, and J. M. Spurgeon, Energy Environ. Sci., 9, 1725 (2016).

21. G. Heremans, C. Trompoukis, N. Daems, T. Bosserez, I. F. J. Vankelecom, J. A. Martens, and J. Rongé, Sustain. Energy Fuels, 1, 2061 (2017).

22. M. Carmo, D. L. Fritz, J. Mergel, and D. Stolten, Int. J. Hydrog. Energy, 38, 4901 (2013).

23. AzurSpace http://www.azurspace.com/index.php/en/products/products-special.

24. M. Bernt and H. A. Gasteiger, J. Electrochem. Soc., 163, F3179 (2016).

25. A. Atifi, H. Mounir, and A. El Marjani, in Renewable and Sustainable Energy Conference (IRSEC), 2014 International, p. 907, IEEE (2014).

26. R. Borup, J. Meyers, B. Pivovar, Y. S. Kim, R. Mukundan, N. Garland, D. Myers, M. Wilson, F. Garzon, and D. Wood, Chem. Rev., 107, 3904 (2007).

27. R. O'Hayre, S.-J. Lee, S.-W. Cha, and F. B. Prinz, J. Power Sources, 109, 483 (2002).

28. D. J. Aiken, Prog. Photovolt. Res. Appl., 8, 563 (2000).

29. K. A. Walczak, J. W. Beeman, and I. D. Sharp, (2018) https://patents.google.com/ patent/WO2018017320A1.

30. S. Hu, N. S. Lewis, J. W. Ager, J. Yang, J. R. McKone, and N. C. Strandwitz, J. Phys. Chem. C, 119, 24201 (2015). 